\title{
Antitumor Effect of Metformin in Combination with Binimetinib on Melanoma Cells
}

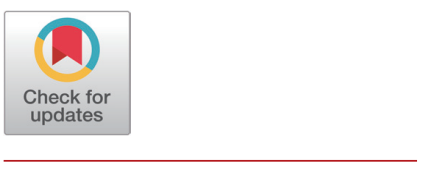

Received: March 26, 2021

Revised: May 4, 2021

Accepted: May 20, 2021

${ }^{\dagger}$ Corresponding author Youngki Lee

Department of Histology, Jeju National University College of Medicine, Jeju

63243, Korea

Tel: +82-64-754-3826

Fax: +82-64-702-2687

E-mail:yklee38@jejunu.ac.kr

Copyright $\odot 2021$ The Korean Society of Developmental Biology.

This is an Open Access article distributed under the terms of the Creative Commons Attribution Non-Commercial License (http://creativecommons.org/licenses/ by-nc/4.0/) which permits unrestricted non-commercial use, distribution, and reproduction in any medium, provided the original work is properly cited.

ORCID

Eunsung Lee

https://orcid.org/0000-0001-8270-0163 Yongjae Kwon

https://orcid.org/0000-0003-0711-9445 Jiwon Kim

https://orcid.org/0000-0002-5984-3219

Deokbae Park

https://orcid.org/0000-0002-0387-8279

Youngki Lee

https://orcid.org/0000-0001-6710-3352

Conflict of interests

The authors declare no potential conflict of interest.

\section{Acknowledgements}

This research was supported by the 2020 scientific promotion program funded by Jeju National University.

Authors' contributions Conceptualization: Lee Y. Data curation: Lee E, Kwon Y Formal analysis: Lee Y, Park D. Methodology: Lee E, Kwon Y.

\author{
Eunsung Lee', Yongjae Kwon', Jiwon Kim', Deokbae Park', and 'Youngki Lee ${ }^{2}$ \\ 'Department of Medicine, Jeju National University College of Medicine, Jeju 63243, Korea \\ ${ }^{2}$ Department of Histology, Jeju National University College of Medicine, Jeju 63243, Korea
}

\begin{abstract}
Cutaneous melanoma is a fatal disease for patients with distant metastasis. Metformin is the most widely used anti-diabetic drug, and proved to suppress cell proliferation and metastasis in diverse cancers including melanoma. We previously reported that MEK inhibitor trametinib increases the expression of epithelial-mesenchymal transition (EMT) regulators and melanoma cell motility, which are suppressed by addition of metformin in A375 melanoma cells. To confirm our findings further, we first evaluated the effect of metformin in combination with another MEK inhibitor binimetinib on cell viability in G361 melanoma cells. We then investigated whether binimetinib affects the expression of EMT regulators and cell motility. We finally monitored the effect of metformin on binimetinib-induced cell migration. Cell viability assay showed that combination index $(\mathrm{Cl})$ value at $\mathrm{ED}_{50}$ is 0.80 , suggesting synergy for the combination of metformin with binimetinib. Our results also revealed that binimetinib increased the expression of EMT regulators such as integrin $\alpha \mathrm{V}$, fibronectin and slug, which correlate well with the enhanced cell migration in wound healing assay. Metformin, on the contrary, suppressed the expression of sparc, integrin $\alpha \mathrm{V}$, fibronectin and $\mathrm{N}$-cadherin with the reduced cell motility. The combination treatment showed that metformin counteracts the binimetinib-induced increase of cell motility. Overall, these results suggest that metformin with binimetinib might be useful as a potential therapeutic adjuvant against cell survival and metastatic activity in melanoma patients.
\end{abstract}

Keywords: Metformin, Binimetinib, Cell viability, Metastasis, Melanoma cell

\section{INTRODUCTION}

Melanoma is a type of skin cancer that develops from malignant transformation of melanocytes. While it is less common than other skin cancers such as basal cell carcinoma and squamous cell carcinoma, melanoma is the deadliest one of skin cancers due to its aggressive spreading ability to other organs if not treated at its early stage. Over the last decade, the annual cases of melanoma increased by nearly 50\%, and International Agency for Research on Cancer (IARC) reported that approximately 287,000 new cases were diagnosed globally in 2018 (Ferlay et al., 2019). Although surgical resection of tumor mass is often curative in the early stage of melanoma progression, melanoma remains one of the cancers resistant to systemic therapy with 5-year survival rate of $25 \%$ for patients with distant 
Software: Kim J, Lee E.

Validation: LeeY.

Writing-original draft: Lee Y.

Writing-review \& editing: Lee Y.

Ethics approval

This article does not require IRB/IACUC approval because there are no human and animal participants. metastasis (American Cancer Society, 2020). However, recent major advancements in molecular targeted and immune-based therapies have made remarkable progress for the treatment of this disease (Domingues et al., 2018).

Comprehensive genetic analysis of cancers have revealed that melanoma has the most frequent mutation rate of all cancers (Alexandrov et al., 2013). In most melanomas, the mitogen-activated protein kinase (MAPK) signaling pathway (also known as RAS/RAF/MEK/ERK pathway) is commonly active through mutations in BRAF (40\%-60\%) and NRAS (15\%-25\%), which eventually lead to uncontrolled cell growth, proliferation and survival (Leonardi et al., 2018). These somatic mutations in melanoma have become principal therapeutic targets and then led to the development of novel targeted drugs, such as inhibitors of BRAF (dabrafenib, vemurafenib and encorafenib) and MEK (trametinib, cobimetinib and binimetinib) (Smalley, 2010). Currently, oncologists typically used the combination of BRAF and MEK inhibitor for the treatment of metastatic BRAF-mutant melanoma (Goyal \& Silberstein, 2015). Despite remarkable advances for the treatment of metastatic melanoma, however, there are significant therapeutic challenges due to acquired resistance or intrinsic unresponsiveness to targeted agents (Holderfield et al., 2014; Long et al., 2014). Moreover, recent studies have reported that MEK inhibition enhances metastatic properties such as cell invasion and migration, which could result in the exasperation of malignancy and subsequent treatment failure (Vultur et al., 2014; Zhao et al., 2017; Lee \& Park, 2021).

Metformin is an oral biguanide agent and has been used as first-line medication for type II diabetes mellitus for a period of over 60 years. It lowers blood glucose levels by reducing hepatic gluconeogenesis and skeletal muscle glucose uptake (Shaw et al., 2005). In addition to its glucoselowering effect, retrospective epidemiological studies have showed that administration of metformin at therapeutic dose reduced the risk of cancer incidence, compared to other antidiabetic medications in diabetic patients (Evans et al., 2005; Bowker et al., 2006). Thereafter, substantial amount of preclinical studies have revealed the anticancer activity of metformin in various types of cancer (Vancura et al., 2018). Several in vivo and in vitro studies by using melanoma cell lines suggested that single agent metformin or combination with BRAF/MEK inhibitors suppress melanoma cell survival and mouse xenograft growth (Niehr et al., 2011; Vujic et al., 2015). Metformin induces cell cycle arrest and apoptosis by interfering with PI3/KmTOR (phosphoinositide 3-kinase/ mammalian target of rapamycin) pathway, a parallel signaling pathway of the MAPK pathway (Woodard \& Platanias, 2010). It also reported to inhibit melanoma cell invasion and metastasis development by suppressing the expression of factors involved in epithelial-mesenchymal transition (EMT) (Cerezo et al., 2013; Tseng et al., 2019).

The present study undertook to investigate whether metformin can potentiate the anticancer effect of MEK inhibitor binimetinib by using melanoma cell line. In addition, our previous report using A375 melanoma cell line showed that MEK inhibitor trametinib increases the expression of EMT regulators and melanoma cell motility, which are suppressed by addition of metformin (Lee \& Park, 2021). A recent report, however, documented that another MEK inhibitor binimetinib inhibited migration capacity of melanoma cells (Ryabaya et al., 2019). In this study, we try to elucidate these differential effects of binimetinib on cell migration further by using G361 melanoma cell. Here we demonstrate that metformin synergistically potentiates the growth inhibitory effect of binimetinib. The present study also shows that binimetinib increases the expression of EMT regulators and enhances cell motility, and metformin counteracts the binimetinib-induced increase of cell motility. These results suggest that metformin with binimetinib might be useful as a potential therapeutic agent against cell survival and metastatic activity in melanoma patients. 


\section{MATERIALS AND METHODS}

\section{Reagents and cell culture}

We purchased the human melanoma cell line G361 from Korean Cell Line Bank (Seoul, Korea). The cells were cultured in RPMI 1640 (Sigma-Aldrich, St. Louis, MO, USA) supplemented with $10 \%(\mathrm{v} / \mathrm{v})$ heat inactivated fetal bovine serum (Gibco BRL, Bethesda, MD, USA) and 1\% streptomycin/penicillin at $37^{\circ} \mathrm{C}$ in a humidified atmosphere consisting of $5 \% \mathrm{CO}_{2}$ and $95 \%$ air. Cells were maintained mycoplasma free by treating $5 \mu \mathrm{g} / \mathrm{mL}$ of Plasmocin (InvivoGen, San Diego, CA, USA). Binietinib (LC Laboratories, Woburn, MA, USA) was initially dissolved in dimethyl sulfoxide (DMSO, Sigma-Aldrich) to a concentration of $1 \mathrm{mM}$ and further diluted in RPMI 1640 media. Metformin (also known as 1,1-dimethylbiguanide hydrochloride) was purchased from Sigma-Aldrich and dissolved in RPMI 1640 media to a working concentration of $100 \mathrm{mM}$. The final concentration of DMSO in the culture media did not exceed $0.1 \%(\mathrm{v} / \mathrm{v})$.

\section{Cell viability assay}

MTT assay was performed to measure cell viability as described previously (Ko et al., 2020). Briefly, cells were harvested and seeded in 24-well plates at a concentration of $5 \times 10^{4}$ cells/well for $24 \mathrm{~h}$. Then, cells were treated with increasing concentrations of binimetinib $(20-80 \mathrm{nM})$, metformin $(0.5-2 \mathrm{mM})$, their combinations or vehicle control for $72 \mathrm{~h}$. Experiments were performed in triplicate, each conducted in quadruplicate. The $\mathrm{IC}_{50}$ values (concentrations of drugs resulting in $50 \%$ decrease in cell viability relative to controls), combination index (CI) and drug reduction index (DRI) were calculated using CompuSyn software (ComboSyn, Paramus, NJ, USA). The CI value is a quantitative measure of the degree of drugs interaction. According to the recommendation of Chou-Talalay (Chou \& Talalay, 1981), CI<1 indicates synergistic effects of drugs; CI=1 indicates additive effect; $\mathrm{CI}>1$ indicates antagonism.

\section{Western blotting}

Western blotting assays were carried out as previously described (Ko et al., 2020). Primary antibodies included ERK, pERK, SPARC, integrin $\alpha \mathrm{V}$, fibronectin, $\mathrm{N}$-cadherin, Slug, $\beta$-actin and GAPDH (all from Santa Cruz Biotechnology, Santa Cruz, CA, USA). Following incubation with secondary antibodies conjugated to horseradish peroxidase (Cell Signaling Technology, Danvers, MA, USA), immunoreactivity was detected with enhanced chemiluminescence method (Santa Cruz Biotechnology).

\section{Colony formation assay}

Cells were plated in 6-well culture dishes at a density of 600 cells per well. After $24 \mathrm{~h}$, cells were treated with metformin, trametinib and their combination. Every three days, medium was changed with fresh medium containing the corresponding concentration of the drugs. Following 12-day treatment, cell colonies were washed with cold PBS and then fixed with ice-cold 100\% methanol. Cells were stained with $0.1 \%$ crystal violet in $20 \%$ methanol for $10 \mathrm{~min}$ and pictures were taken with a digital camera (Olympus Korea, Seoul, Korea).

\section{Wound healing assay}

G361 melanoma cells were grown to $80 \%$ confluence in 12 -well culture plates in complete medium. After serum-starving cells for $24 \mathrm{~h}$, a $200-\mu \mathrm{L}$ tip was used to create a consistent scratch in the cell monolayer. All wells were then carefully washed with culture medium and drug solutions in complete medium were added and incubated for 24 or $48 \mathrm{~h}$. Photographs were taken with an 
Olympus 1×70 microscope and an Olympus DP72 camera and DPController Software (Olympus Korea).

\section{Statistical analysis}

Data are expressed in the form of mean $\pm \mathrm{SE}$. The statistical analysis was done by a one-way ANOVA. Differences between means in each analysis were considered statistically significant when yielding $p \leq 0.05$.

\section{RESULTS}

\section{Combination of metformin with binimetinib shows synergistic growth inhibitory effect in G361 melanoma cells}

To assess the response of G361 melanoma cells to treatment with binimetinib, metformin and their combination, we first carried out MTT cell viability assays. G361 cells were treated with varying concentrations of drugs as single agent or their combination for $72 \mathrm{~h}$. As shown Fig. 1A, relative cell viability was reduced following treatment with binimetinib and metformin in a dosedependent manner. $\mathrm{IC}_{50}$ values (concentrations of drugs resulting in $50 \%$ decrease in cell viability relative to controls) for binimetinib and metformin were $40.3 \mathrm{nM}$ and $1.53 \mathrm{mM}$, respectively.

We then analyzed the growth inhibitory effect by combining metformin with binimetinib in constant ratios to each other. To quantify the effects of drug combination, we used the CompuSyn software to obtain the $\mathrm{CI}$ value for each combination therapy. The $\mathrm{CI}$ values in combination at low doses ranged from 0.89 to 1.03 indicating synergism or additivity according to the method of Chou-Talalay (Chou \& Talalay, 1981). In contrast, CI value at high dose (at $2 \mathrm{mM}$ of metformin and $50 \mathrm{nM}$ binimetinib) was 1.18 denoting antagonistic effect between two drugs (Fig. 1B). However, $\mathrm{CI}$ value at $\mathrm{ED}_{50}$ (the dose effecting $50 \%$ of growth inhibition compared to control) was 0.80 , showing synergism between two drugs.

We previously suggested that ERK activation in response to metformin serves as a potential predictive biomarker in the combination therapy by using metformin with other targeted agent (Lee \& Park, 2021). Therefore, we performed western blotting for ERK activation following treatments with metformin and binimetinib. As shown in Fig. 1C, treatments with metformin and binimetinib suppressed the level of pERK in dose-dependent manner.

\section{Effects of metformin treatment with binimetinib on colony formation}

Our present cell viability assay showed that combinatory treatment of metformin with binimetinib synergistically inhibits the survival of G361 melanoma cells. To extend these cell survival results into long-term effect of the combination treatment, we next performed colony formation assay by culturing G361 melanoma cells for 12 days. As shown in Fig. 2, treatment with metformin or binimetinib as single agent led to a partial inhibition of colony formation, while the combination treatment potentiated the inhibitory effect on the formation and growth of cell colonies as compared to either agent alone. These results further confirmed the synergistic inhibitory effect of metformin with binimetinib on cell survival analyzed by using MTT assay.

\section{Effects on expression of factors involved in EMT following treatment with metformin and binimetinib}

Several research groups documented that metformin suppresses cancer cell motility and metastasis development (Cerezo et al., 2013; Tseng et al., 2019). On the other hand, recent studies 

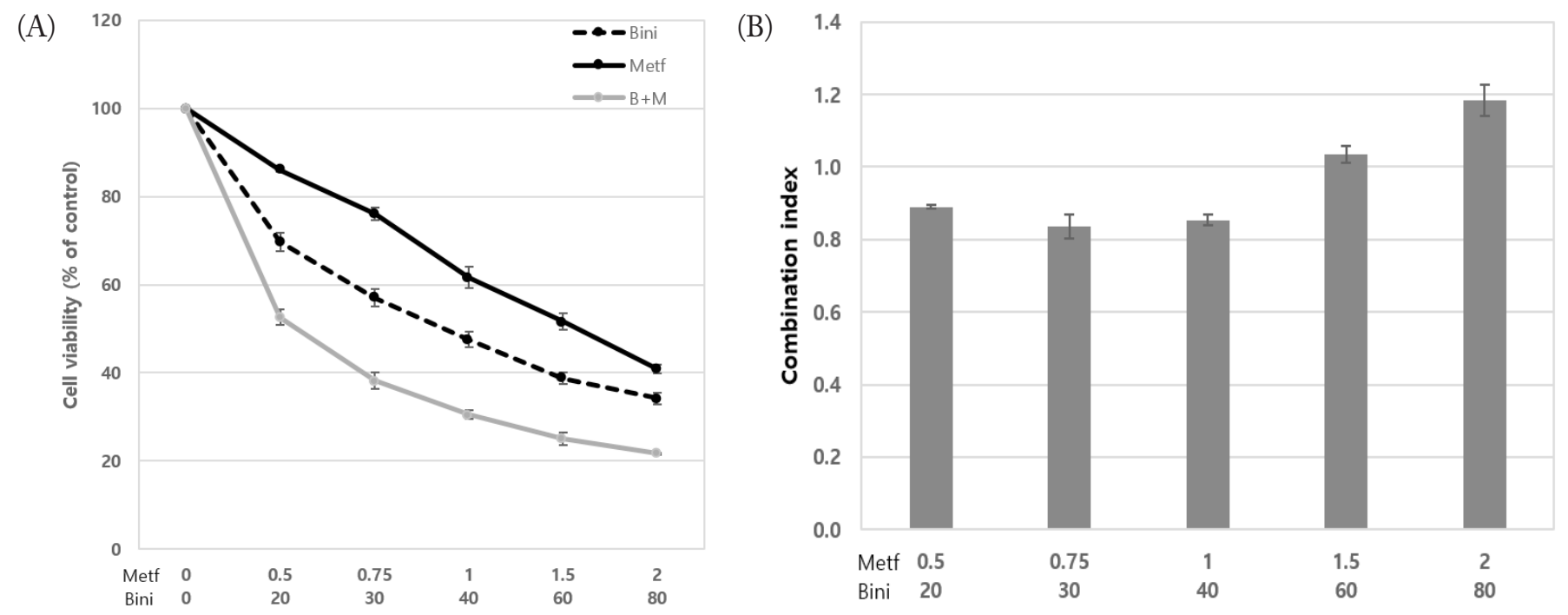

(C)

\section{Metformin (mM)}

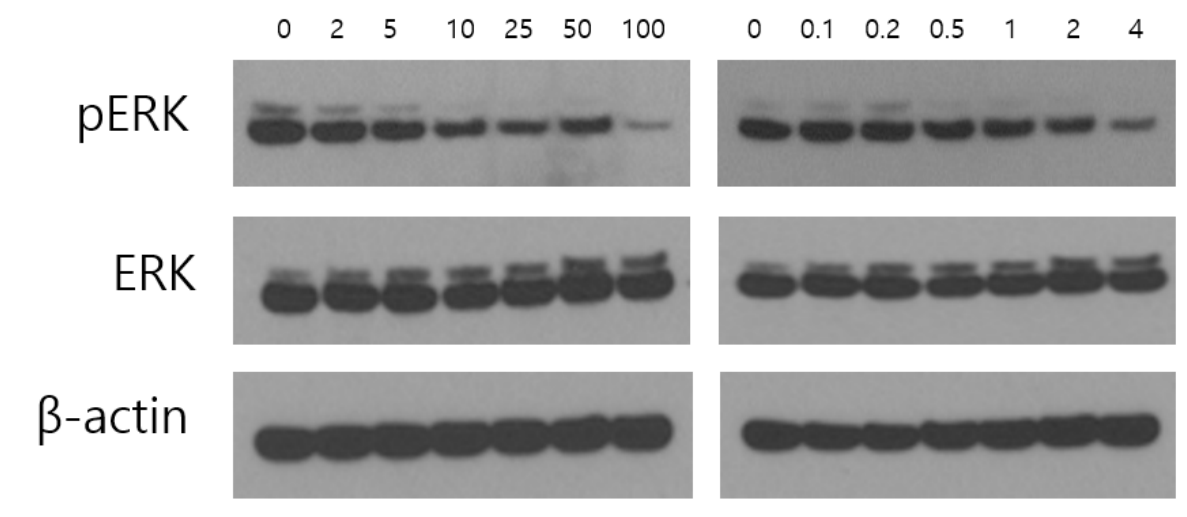

Fig. 1. Combined treatment of metformin with binimetinib has synergistic effect on cell viability in G361 cells. (A) Dose-response curves for metformin (Metf), binimetinib (Bini) and their combination $(B+M)$ in constant ratio $(40: 1)\left(n=3\right.$, error bar represents SE). G361 cells were seeded at $5 \times 10^{4}$ cells/ well $(0.5 \mathrm{~mL})$ in 24-well culture plates, incubated for $24 \mathrm{~h}$ and then treated with metformin, binimetinib and their combination for $72 \mathrm{~h}$. MTT assay was performed for the determination of cell viability. The viability of control cells was regarded as $100 \%$. (B) Combination index $(\mathrm{Cl})$ values for the various combination points of metformin and trametinib. The $\mathrm{Cl}$ values were calculated by using CompuSyn software. (C) Western blot analysis for the activity of ERK. Cells were treated with increasing concentrations of metformin and binimetinib for $24 \mathrm{~h}$. $\beta$-actin was used as a loading control.

including us have revealed that MEK inhibition increases metastatic properties in melanoma and breast cancer cells, which could result in the deterioration of malignancy and treatment failure (Vultur et al., 2014; Zhao et al., 2017; Lee \& Park, 2021). To confirm this issue further, we performed western blot assay using G361 melanoma cells to determine whether metformin and binimetinib affect the expression of factors involved in EMT. As shown in Fig. 3, treatment with binimetinib increased the expression level of integrin $\alpha \mathrm{V}$, fibronectin and slug, and exerted little effect on the expression of sparc and $\mathrm{N}$-cadherin. In contrast, metformin remarkably decreased the levels of sparc, integrin $\alpha \mathrm{V}$, fibronectin and $\mathrm{N}$-cadherin in dose-dependent manner.

\section{Effects of cell migration following treatment with metformin, binimetinib and their combination}

Next, we wished to determine whether the molecular changes found in the expression of EMT regulators following treatment with metformin and binimetinib lead to functional alteration, such 
Binimetinib (nM)
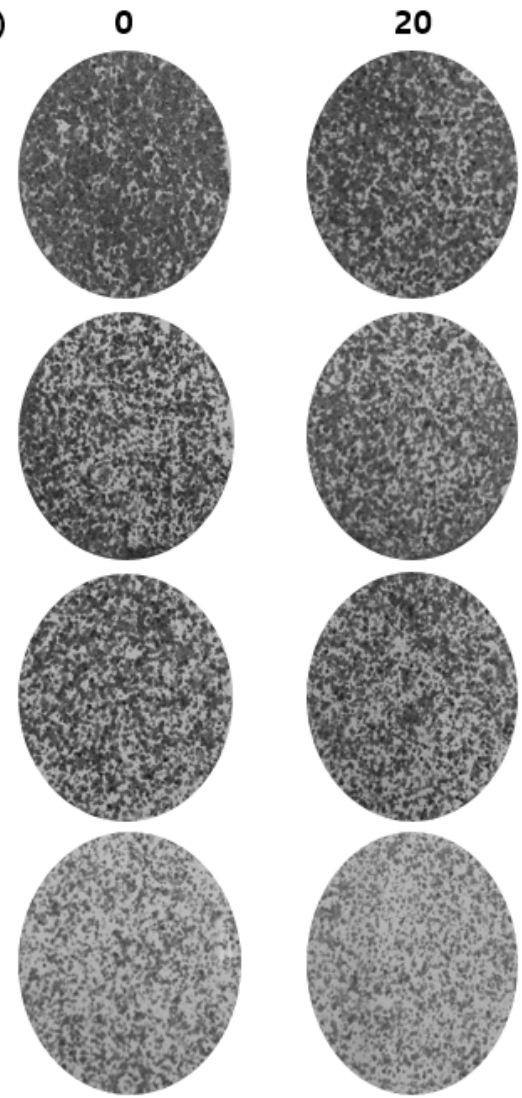

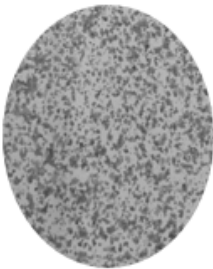

20
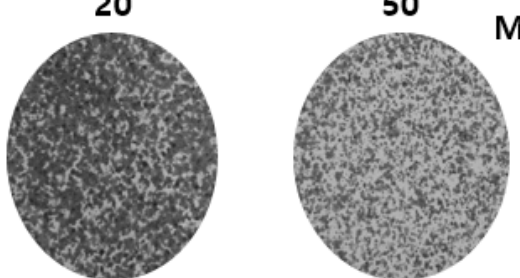

Metformin $(\mathrm{mM})$

0

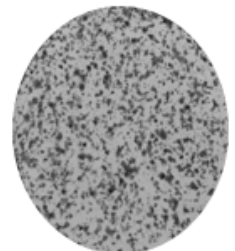

0.25
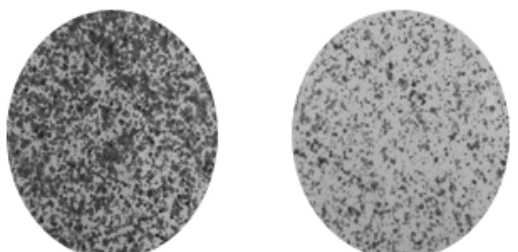

0.5

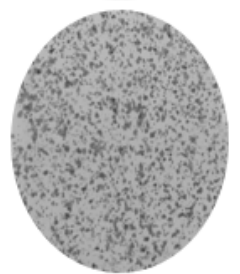

Fig. 2. Effect of metformin and binimetinib combination on colony formation in G361 cells. Cells were seeded in 6-well plates at a density of 300 cells per well. At $24 \mathrm{~h}$ after plating, cells were treated with indicated concentrations of metformin and/or binimetinib. Following 12-day treatment, cell colonies were stained using crystal violet dye and pictures were taken with a digital camera.

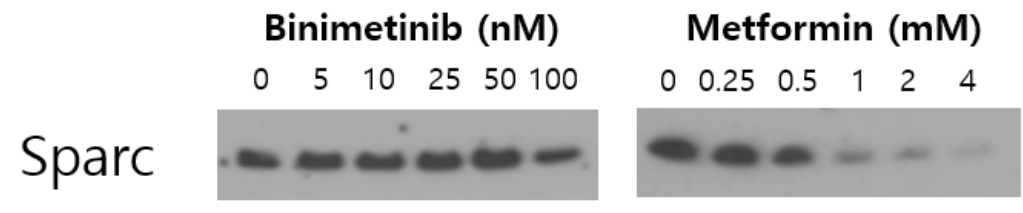

\section{Integrin $\alpha \mathrm{V}$}
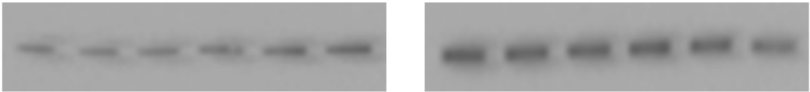

\section{Fibronectin}
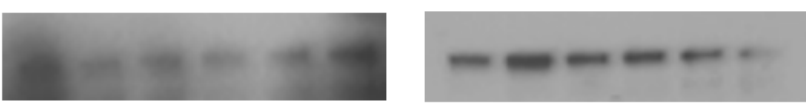

$\mathrm{N}$-cadherin
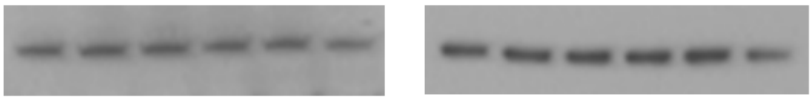

\section{Slug}
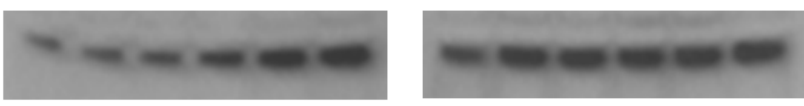

\section{GAPDH}
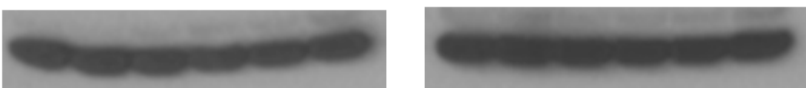

Fig. 3. Western blot analysis for EMT markers after 24-h treatment. Cells were treated with increasing doses of metformin or binimetinib for $24 \mathrm{~h}$. Glyceraldehyde-3-phosphate dehydrogenase (GAPDH) was used as a loading control. 
as cell motility. For this purpose, we monitored cell migration in G361 cells using wound healing assay. Low doses of binimetinib $(5-50 \mathrm{nM})$ promoted cell migration but showed little effect at high dose (100 nM) (Fig. 4A). In contrast, treatment with low doses of metformin (0.25-1 mM) alone neither enhanced nor inhibited cell migration compared to control, but high doses of metformin (2-4 mM) inhibited cell migration (Fig. 4B). Combined treatments of metformin with binimetinib suppressed the binimetinib-induced cell migration (Fig. 4C).

\section{DISCUSSION}

Molecular targeted therapy against cutaneous melanoma with BRAF mutation has shown a remarkable progress with the advent of small molecule inhibitors of BRAF itself or its downstream effector MEK (Smalley, 2010; Domingues et al., 2018). Combined treatment of BRAF inhibitor (dabrafenib or vemurafenib) with MEK inhibitor (trametinib) proved to display clinical benefits, such as better overall response rate and progression free survival, than each monotherapy (Long et al., 2018). However, half of patients treated with the combination therapy eventually experience fatal progression of their disease at 9-10 months following initial treatment, mainly due to drug resistance with severe toxicities (Long et al., 2014; Welsh et al., 2016). One of the mechanistic factors underlying the drug resistance arising from the combination therapy of BRAF and MEK

(A)

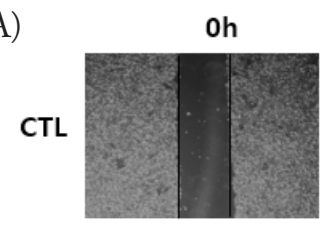

5

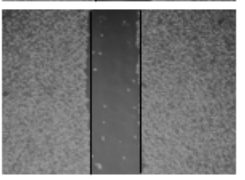

10

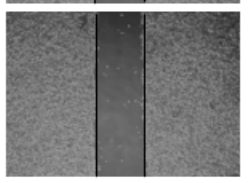

25

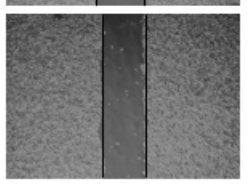

50

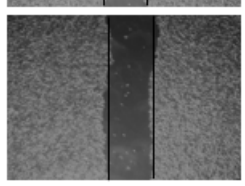

100

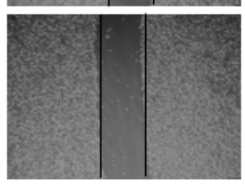

$24 \mathrm{~h}$
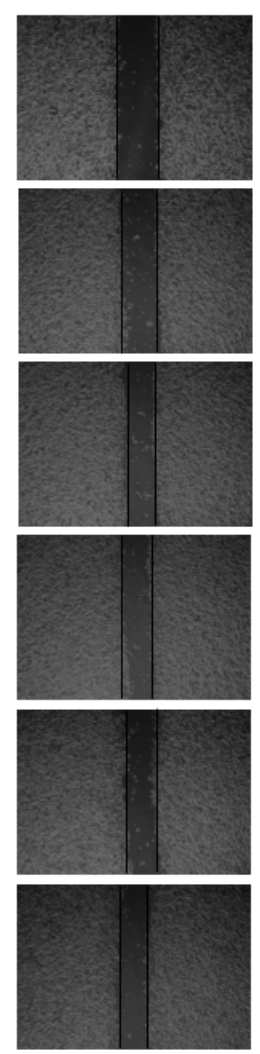

$48 \mathrm{~h}$
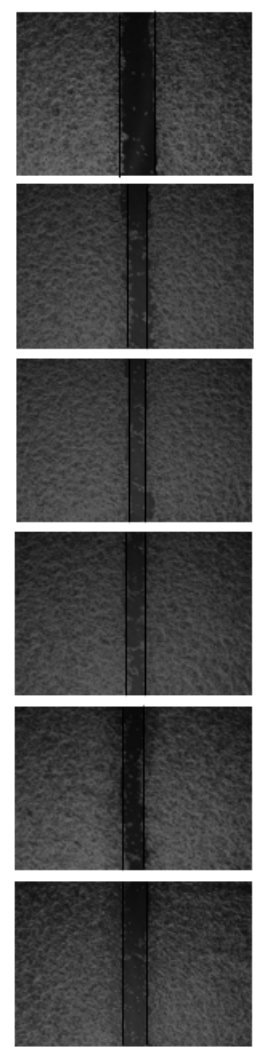

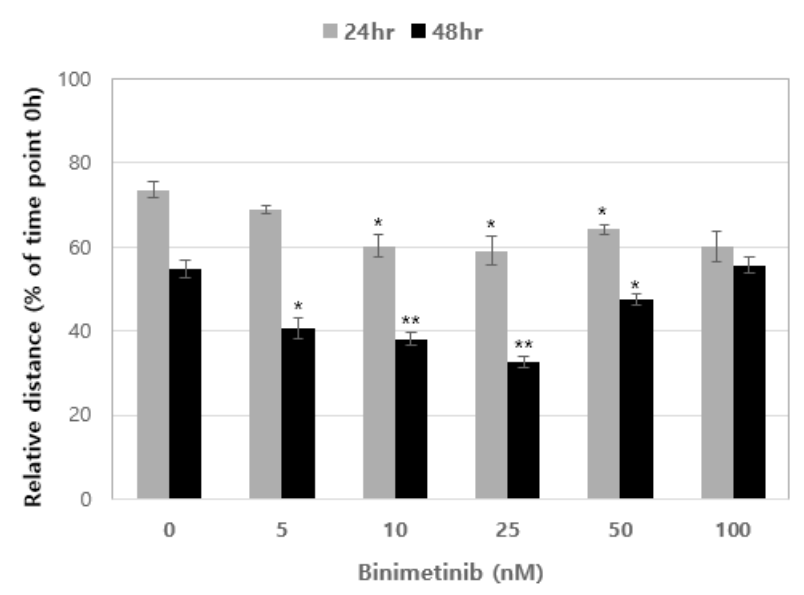

Binimetinib (nM)

Fig. 4. Wound healing assay to determine the effects of binimetinib (A), metformin (B) or their combination (C) on cell migration in G361 cells. Representative photographs taken at $0 \mathrm{~h}, 24 \mathrm{~h}$, and $48 \mathrm{~h}$ are shown. Bar graphs show the relative gap distance against controls of time point zero. (A), (B) Cells were treated with increasing concentrations of binimetinib or metformin. (C) Cells were treated with binimetinib (25 nM), metformin (2 mM) or their combination. $\mathrm{n}=3$, error bars represents SE. * $p<0.05,{ }^{* *} p<0.01$. 
(B)

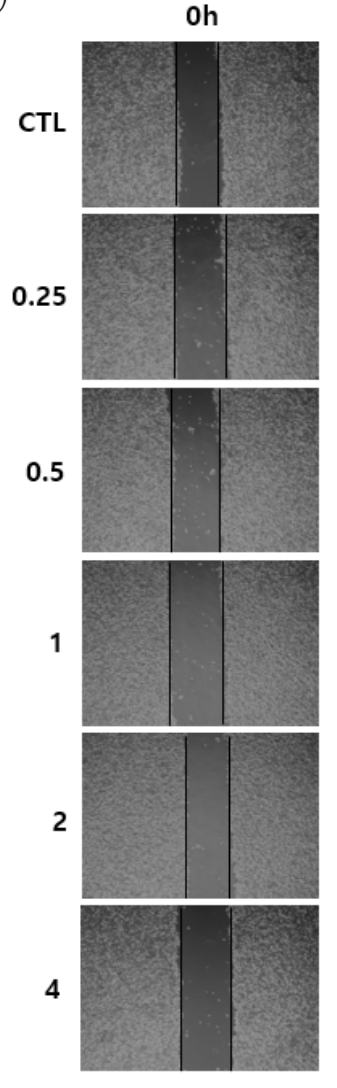

$24 \mathrm{~h}$
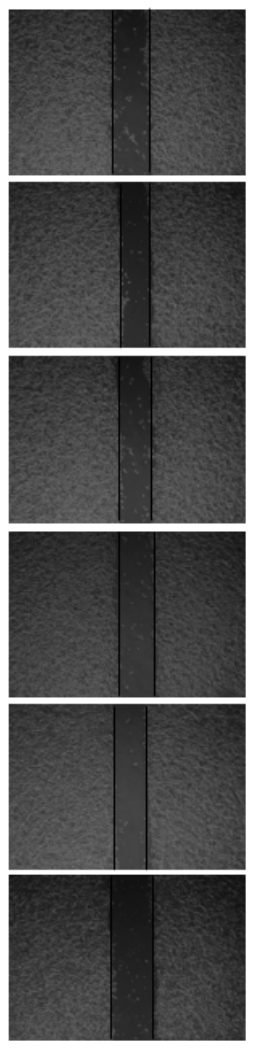

$48 \mathrm{~h}$
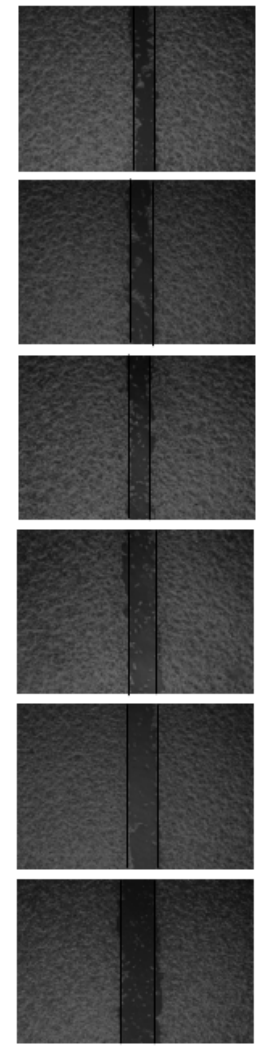
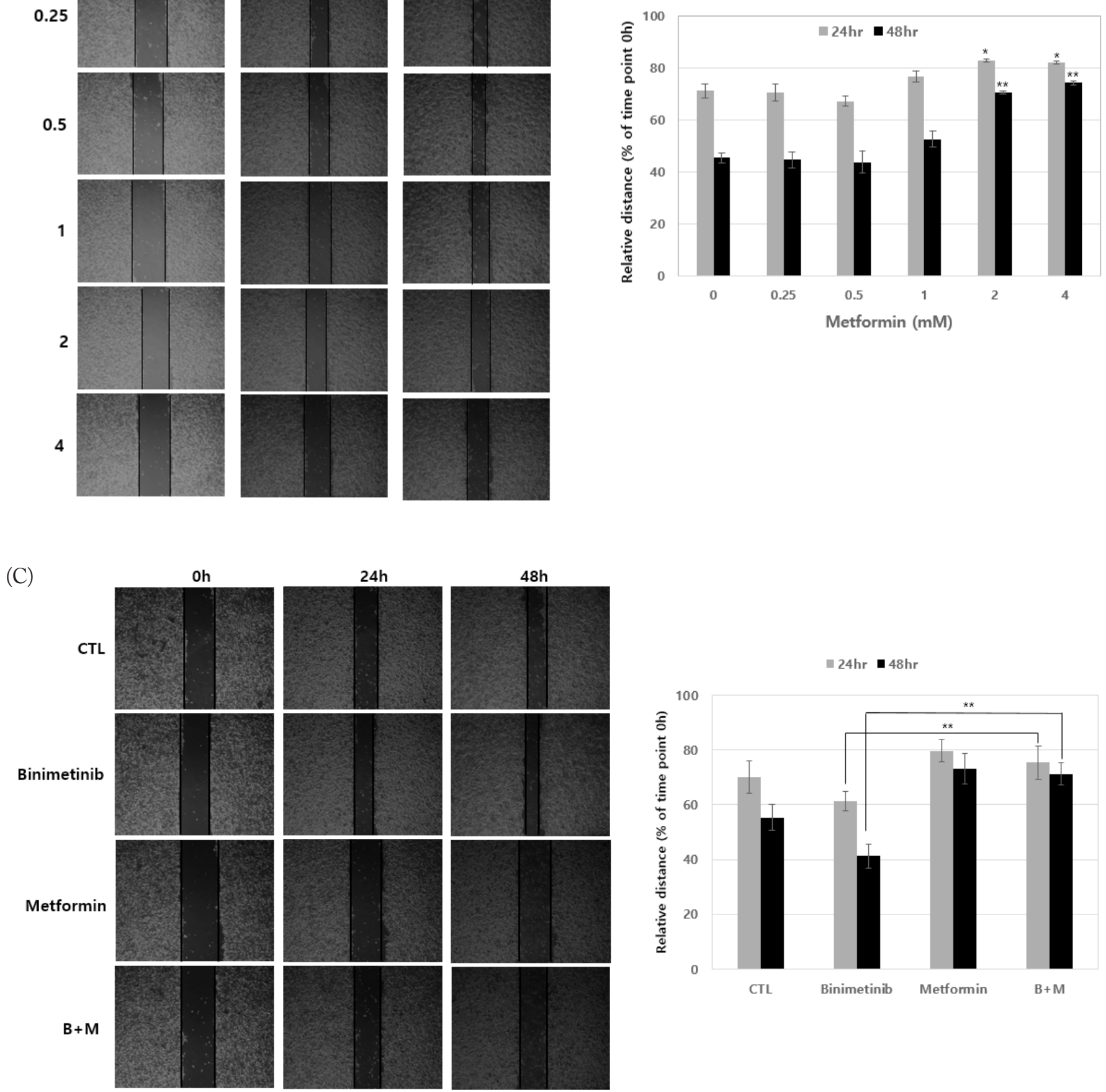

$B+M$
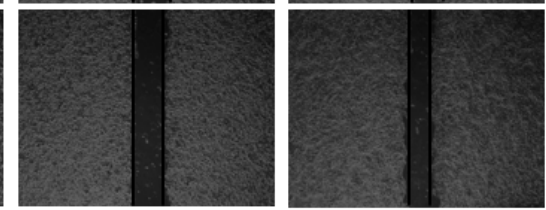

Metformin
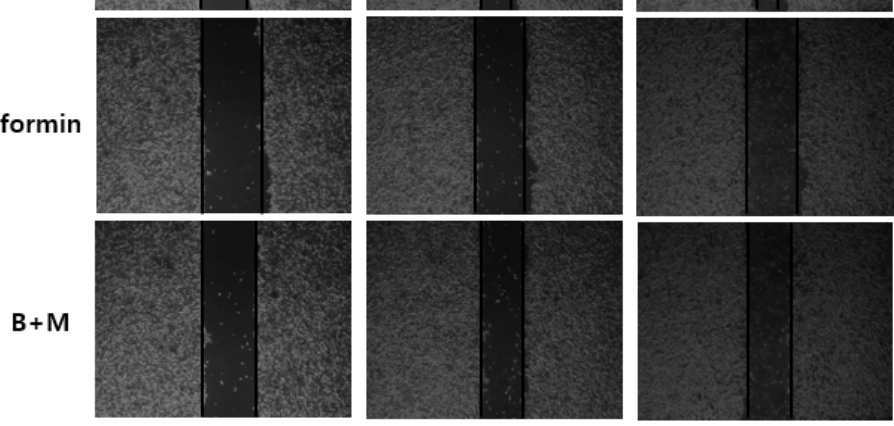

Fig. 4. Continued. 
inhibitors is the compensatory activation of parallel signaling cascades such as $\mathrm{PI} 3 \mathrm{~K} / \mathrm{mTOR}$ pathway (Welsh et al., 2016). Another therapeutically important problem is that MEK inhibition reported to enhance metastatic activities such as cell migration and invasion in melanoma and breast cancer cells (Vultur et al., 2014; Zhao et al., 2017). Thus, there remains an unmet urgent need for the development of novel therapeutic strategy with improvement of clinical efficacy by overcoming resistance as well as concomitant reductions of side effects.

The first goal of this preclinical study was to develop rational combination therapy for the treatment of melanoma by combining metformin and binimetinib. Metformin as antidiabetic drug has been known to be a well-tolerated drug with safety profile and little drug resistance (Scherbakov et al., 2016). It inhibits cell proliferation through activation of AMP-activated protein kinase (AMPK), which could suppress MEK inhibition-induced compensatory activation of PI3K/ mTOR signaling pathway (Woodard \& Platanias, 2010). Thus, many research groups anticipated that the combination of metformin with MEK inhibitor would give synergistic antitumor activity through simultaneous inhibition of MAPK and PI3K/mTOR pathways. However, the results showed divergent effects of the combination therapy on melanoma cell viability (Vujic et al., 2015). In our previous studies, we suggested that the combination of metformin with trametinib shows the differential growth inhibitory effect; synergistic effects in cell line in which metformin suppresses ERK activity but antagonistic effect in cell line with metformin-induced ERK activation (Lee \& Park, 2021). Indeed, the effect of metformin on ERK activity has shown conflicting results in various types of cancer cells (Martin et al., 2012; Morgillo et al., 2013; Della Corte et al., 2016). The present study showed that metformin suppresses ERK activation dose dependently and the combined treatment of metformin with binimetinib leads to synergistic growth inhibition of G361 melanoma cells. These results confirm further our suggestion that ERK activity in response to metformin could serve as a predictive biomarker for development of effective therapeutic regimens when using metformin in combination with other anticancer agents.

While MEK inhibition leads to inhibition of cell proliferation through suppression of MAPK signaling pathway, recent studies reported that it could induce metastatic properties such as migration and invasion (Vultur et al., 2014; Zhao et al., 2017). Our previous study also showed that MEK inhibition with trametinib increases the expression of core EMT regulators such as fibronectin, twist1 and slug in A375 melanoma cells and induces cell invasion and migration (Lee \& Park, 2021). In the present study, we tried to confirm this issue again by employing binimetinib, another MEK inhibitor, in G361 melanoma cells. Our present data showed that binimetinib also increases the levels of EMT regulators, and these molecular changes functionally well correlated with the increase of melanoma cell migration. On the contrary, metformin remarkably reduced EMT regulators, which is in agreement with previous reports by other research groups (Cerezo et al., 2013; Tseng et al., 2019). Undoubtedly, the increased metastatic behavior in response to MEK inhibition could be therapeutically a serious obstacle to the effective treatment of melanoma. Thus, we extended the findings to the combination of metformin with binimetinib, and found that metformin counteracts the pro-migratory activity of binimetinib. These findings support the premise that metformin might be useful as a potential anticancer agent for melanoma patients via not only suppression of cell proliferation but also inhibition of metastasis.

Our finding that binimetinib leads to enhancement of cell migration is inconsistent with the report of Ryabaya et al. (2019) in which binimetinib rather inhibits melanoma cell migration. The reason for this apparent contradiction between two studies is not clear, but we suppose that the discrepancy might reside in different treatment regimens of binimetinib. They used binimetinib at $2 \mu \mathrm{M}$, whereas we applied the increasing concentrations of binimetinib from $5 \mathrm{nM}$ up to $100 \mathrm{nM}$. Moreover, we found that binimetinib at low doses $(5-25 \mathrm{nM})$ enhances cell migration in dose- 
dependent manner, then retards the extent of cell migration at $50 \mathrm{nM}$, and does not affect cell migration at $100 \mathrm{nM}$. These results suggest that inhibition of cell migration following treatment with $2 \mu \mathrm{M}$ of binimetinib could result from apoptosis, which they showed by flow cytometry analysis using Annexin V/PI double staining and increased level of cleaved PARP in Western blot. Further studies by using the same melanoma cell line will be needed to elucidate this issue.

In summary, the present preclinical study shows the first in vitro results that combination of metformin with binimetinib might be a promising therapeutic option for treatment of patients with melanoma. In particular, our data support our previous suggestion that differential alteration of ERK activity in response to metformin could serve as a predictive biomarker for the efficacy of metformin monotherapy or combination therapies with other antitumor drugs. Further in vivo studies using mouse xenograft models and clinical trials are required to better elucidate the combined effect of metformin with binimetinib on antitumor activity in melanoma.

\section{REFERENCES}

Alexandrov LB, Nik-Zainal S, Wedge DC, Aparicio SAJR, Behjati S, Biankin AV, Bignell GR, Bolli N, Borg A, Børresen-Dale AL, Boyault S, Burkhardt B, Butler AP, Caldas C, Davies HR, Desmedt C, Eils R, Eyfjörd JE, Foekens JA, Greaves M, Hosoda F, Hutter B, Ilicic T, Imbeaud S, Imielinski M, Jäger N, Jones DTW, Jones D, Knappskog S, Kool M, Lakhani SR, López-Otín C, Martin S, Munshi NC, Nakamura H, Northcott PA, Pajic M, Papaemmanuil E, Paradiso A, Pearson JV, Puente XS, Raine K, Ramakrishna M, Richardson AL, Richter J, Rosenstiel P, Schlesner M, Schumacher TN, Span PN, Teague JW, Totoki Y, Tutt ANJ, ValdésMas R, van Buuren MM, van 't Veer L, Vincent-Salomon A, Waddell N, Yates LR, Australian Pancreatic Cancer Genome Initiative, ICGC Breast Cancer Consortium, ICGC MMMLSeq Consortium, ICGC PedBrain, Zucman-Rossi J, Andrew Futreal P, McDermott U, Lichter P, Meyerson M, Grimmond SM, Siebert R, Campo E, Shibata T, Pfister SM, Campbell PJ, Stratton MR (2013) Signatures of mutational processes in human cancer. Nature 500:415-421.

American Cancer Society (2020) Cancer facts \& figures 2020. Available from: https://www.cancer. $\mathrm{org} /$ research/cancer-facts-statistics/all-cancer-facts-figures/cancer-facts-figures-2020.html. Accessed at Feb 5, 2021.

Bowker SL, Majumdar SR, Veugelers P, Johnson JA (2006) Increased cancer-related mortality for patients with type 2 diabetes who use sulfonylureas or insulin. Diabetes Care 29:254-258.

Cerezo M, Tichet M, Abbe P, Ohanna M, Lehraiki A, Rouaud F, Allegra M, Giacchero D, Bahadoran P, Bertolotto C, Tartare-Deckert S, Ballotti R, Rocchi S (2013) Metformin blocks melanoma invasion and metastasis development in AMPK/p53-dependent manner. Mol Cancer Ther 12:1605-1615.

Chou TC, Talalay P (1981) Generalized equations for the analysis of inhibitions of MichaelisMenten and higher-order kinetic systems with two or more mutually exclusive and nonexclusive inhibitors. Eur J Biochem 115:207-216.

Della Corte CM, Ciaramella V, Di Mauro C, Castellone MD, Papaccio F, Fasano M, Sasso FC, Martinelli E, Troiani T, De Vita F, Orditura M, Bianco R, Ciardiello F, Morgillo F (2016) Metformin increases antitumor activity of MEK inhibitors through GLI1 downregulation in LKB1 positive human NSCLC cancer cells. Oncotarget 7:4265-4278.

Domingues B, Lopes JM, Soares P, Pópulo H (2018) Melanoma treatment in review. ImmunoTargets Ther 7:35-49.

Evans JMM, Donnelly LA, Emslie-Smith AM, Alessi DR, Morris AD (2005) Metformin and 
reduced risk of cancer in diabetic patients. Br Med J 330:1304-1305.

Ferlay J, Colombet M, Soerjomataram I, Mathers C, Parkin DM, Piñeros M, Znaor A, Bray F (2019) Estimating the global cancer incidence and mortality in 2018: GLOBOCAN sources and methods. Int J Cancer 144:1941-1953.

Goyal G, Silberstein PT (2015) Systemic therapy in metastatic melanoma. Fed Pract 32:57S-65S.

Holderfield M, Deuker MM, McCormick F, McMahon M (2014) Targeting RAF kinases for cancer therapy: BRAF-mutated melanoma and beyond. Nat Rev Cancer 14:455-467.

Ko E, Baek S, Kim J, Park D, Lee Y (2020) Antitumor activity of combination therapy with metformin and trametinib in non-small cell lung cancer cells. Dev Reprod 24:113-123.

Lee Y, Park D (2021) Effect of metformin in combination with trametinib and paclitaxel on cell survival and metastasis in melanoma cells. Anticancer Res 41:1387-1399.

Leonardi GC, Falzone L, Salemi R, Zanghi A, Spandidos DA, Mccubrey JA, Candido S, Libra M (2018) Cutaneous melanoma: From pathogenesis to therapy (review). Int J Oncol 52:10711080.

Long GV, Eroglu Z, Infante J, Patel S, Daud A, Johnson DB, Gonzalez R, Kefford R, Hamid O, Schuchter L, Cebon J, Sharfman W, McWilliams R, Sznol M, Redhu S, Gasal E, Mookerjee B, Weber J, Flaherty KT (2018) Long-term outcomes in patients with BRAF V600-mutant metastatic melanoma who received dabrafenib combined with trametinib. J Clin Oncol 36:667-673.

Long GV, Fung C, Menzies AM, Pupo GM, Carlino MS, Hyman J, Shahheydari H, Tembe V, Thompson JF, Saw RP, Howle J, Hayward NK, Johansson P, Scolyer RA, Kefford RF, Rizos H (2014) Increased MAPK reactivation in early resistance to dabrafenib/trametinib combination therapy of BRAF-mutant metastatic melanoma. Nat Commun 5:5694.

Martin MJ, Hayward R, Viros A, Marais R (2012) Metformin accelerates the growth of BRAFV600E-driven melanoma by upregulating VEGF-A. Cancer Discov 2:344-355.

Morgillo F, Sasso FC, Della Corte CM, Vitagliano D, D’Aiuto E, Troiani T, Martinelli E, De Vita F, Orditura M, De Palma R, Ciardiello F (2013) Synergistic effects of metformin treatment in combination with gefitinib, a selective EGFR tyrosine kinase inhibitor, in LKB1 wild-type NSCLC cell lines. Clin Cancer Res 19:3508-3519.

Niehr F, von Euw E, Attar N, Guo D, Matsunaga D, Sazegar H, Ng C, Glaspy JA, Recio JA, Lo RS, Mischel PS, Comin-Anduix B, Ribas A (2011) Combination therapy with vemurafenib (PLX4032/RG7204) and metformin in melanoma cell lines with distinct driver mutations. J Transl Med 9:76.

Ryabaya O, Prokofieva A, Akasov R, Khochenkov D, Emelyanova M, Burov S, Markvicheva E, Inshakov A, Stepanova E (2019) Metformin increases antitumor activity of MEK inhibitor binimetinib in 2D and 3D models of human metastatic melanoma cells. Biomed Pharmacother 109:2548-2560.

Scherbakov AM, Sorokin DV, Tatarskiy Jr. VV, Prokhorov NS, Semina SE, Berstein LM, Krasil'nikov MA (2016) The phenomenon of acquired resistance to metformin in breast cancer cells: The interaction of growth pathways and estrogen receptor signaling. IUBMB Life 68:281-292.

Shaw RJ, Lamia KA, Vasquez D, Koo SH, Bardeesy N, Depinho RA, Montminy M, Cantley LC (2005) The kinase LKB1 mediates glucose homeostasis in liver and therapeutic effects of metformin. Science 310:1642-1646.

Smalley KS (2010) PLX-4032, a small-molecule B-Raf inhibitor for the potential treatment of malignant melanoma. Curr Opin Invest Drugs 11:699-706.

Tseng HW, Li SC, Tsai KW (2019) Metformin treatment suppresses melanoma cell growth and 
motility through modulation of microRNA expression. Cancers 11:209.

Vancura A, Bu P, Bhagwat M, Zeng J, Vancurova I (2018) Metformin as an anticancer agent. Trends Pharmacol Sci 39:867-878.

Vujic I, Sanlorenzo M, Posch C, Esteve-Puig R, Yen AJ, Kwong A, Tsumura A, Murphy R, Rappersberger K, Ortiz-Urda S (2015) Metformin and trametinib have synergistic effects on cell viability and tumor growth in NRAS mutant cancer. Oncotarget 6:969-978.

Vultur A, Villanueva J, Krepler C, Rajan G, Chen Q, Xiao M, Li L, Gimotty PA, Wilson M, Hayden J, Keeney F, Nathanson KL, Herlyn M (2014) MEK inhibition affects STAT3 signaling and invasion in human melanoma cell lines. Oncogene 33:1850-1861.

Welsh SJ, Rizos H, Scolyer RA, Long GV (2016) Resistance to combination BRAF and MEK inhibition in metastatic melanoma: Where to next? Eur J Cancer 62:76-85.

Woodard J, Platanias LC (2010) AMP-activated kinase (AMPK)-generated signals in malignant melanoma cell growth and survival. Biochem Biophys Res Commun 398:135-139.

Zhao Y, Ge CC, Wang J, Wu XX, Li XM, Li W, Wang SS, Liu T, Hou JZ, Sun H, Fang D, Xie SQ (2017) MEK inhibitor, PD98059, promotes breast cancer cell migration by inducing $\beta$-catenin nuclear accumulation. Oncol Rep 38:3055-3063. 